\title{
Editorial: A look back and to the future with classic papers in theoretical ecology
}

\section{Alan Hastings ${ }^{1}$}

Received: 8 August 2019 / Accepted: 8 August 2019 /Published online: 20 August 2019

(C) Springer Nature B.V. 2019

The first paper in this issue, by Gilbert and Yeakel, is the first in what will be an ongoing series of papers in Theoretical Ecology revisiting an impactful paper in theoretical ecology and explaining how it relates to current and future research. This paper focuses on Levin's classic 1974 paper on spatial ecology. More papers of a similar nature will be appearing regularly in Theoretical Ecology. This ongoing series should be of special interest for younger researchers to see how important themes in theoretical ecology have arisen and how these ideas continue to play a central role in understanding ecological questions. Suggestions for future subjects in this series are welcome.

Alan Hastings

Editor-in-Chief

Alan Hastings

amhastings@ucdavis.edu

1 Department of Environmental, Science and Policy, University of California, One Shields Avenue, Davis, CA 95616, USA 